\title{
HÁBITAT Y VULNERABILIDAD, REFLEXIONES DESDE LO CONCEPTUAL
}

\author{
WILLIAMS GILBERTO JIMÉNEZ GARCÍA
}

Recibido el 9 de agosto de 2012 y aprobado el 25 de abril de 2013

\section{RESUMEN}

Los esfuerzos por generar conceptos claros y precisos acerca de vulnerabilidad y hábitat, han hecho que estos conceptos pierdan claridad y se simplifiquen; a pesar de su importancia en la vida del hombre, se ha perdido el sustento de los mismos y se han reducido a "supuestos", que son usados en el lenguaje común y, a veces, de manera inapropiada. El presente escrito tiene como objetivo reflexionar acerca de la vulnerabilidad y el hábitat como conceptos holísticos e integrales. Para tal fin se realizó una revisión documental de varios textos científicos y se plantearon algunos modelos explicativos para interpretarlos, concluyendo que estos conceptos se deben comprender a través de su interrelación en la construcción social del territorio.

\section{PALABRAS CLAVE}

Hábitat, vulnerabilidad, complejidad, sistemas, interrelaciones.

\section{HABITAT AND VULNERABILITY, REFLECTIONS FROM THE CONCEPTUAL SIDE}

\begin{abstract}
The efforts to generate clear and precise concepts about vulnerability and habitat have resulted in the simplification and loss of clarity of these concepts. Despite their importance to human life, they have lost support and have been reduced to "assumptions" that are used in common parlance and sometimes inappropriately. The main objective of this article is to reflect on vulnerability and habitat as holistic and integrated concepts. For this purpose a literature review of various scientific texts was carried out and some explanatory models to interpret it were proposed, concluding that these concepts must be understood through their interrelation in the social construction of territory.
\end{abstract}

\section{KEYWORDS}

Habitat, vulnerability, vulnerability factors, complexity, systems, interrelations.

\section{EL HÁBITAT, UN ESCENARIO VULNERABLE}

La sociedad actual se encuentra en una encrucijada que la reúne a una novedosa voluntad por agregar nuevos temas y redefinir conceptos que permitan comprender y analizar integralmente una realidad, hasta el momento muy definida, pero poco interpretada. Los esfuerzos por construir 
nuevos paradigmas de hábitat y vulnerabilidad que contribuyan a la construcción de imaginarios colectivos que sustenten la acción de las personas, la continuidad de los procesos sociales, el intercambio y construcción de información, y la supervivencia del planeta a escala global en medio de una virtualización de la realidad y una lucha cada vez más evidente por los recursos naturales, son la manifestación de una nueva forma de ver el mundo.

Es necesario reconocer la relación existente en la construcción de los conceptos de hábitat y vulnerabilidad a partir de las relaciones estructurales que el ser humano teje con su entorno. En otras palabras, reconocer que el hábitat y la vulnerabilidad influyen de manera directa -y son influidos- en aspectos sociales, tales como la construcción de identidad y la convivencia en condiciones de equidad y seguridad; asimismo, en aspectos ambientales, tales como la seguridad física que ofrecen los entornos naturales, la oferta y la demanda de los recursos naturales y el diseño y producción de espacios dignos para vivir.

La anterior reflexión se enmarca en un escenario que permite integrar a la vulnerabilidad y el hábitat en una problemática real -no solo conceptualmente-, donde el hábitat contiene las amenazas a las que son vulnerables las poblaciones. Por lo tanto, se puede argumentar que existe una relación directa entre la forma en cómo los pobladores construyen su hábitat y las situaciones que condicionan esta construcción, mediada en ocasiones por el grado de exposición de estos pobladores a amenazas naturales, socio-naturales o antrópicas, las decisiones políticas de comunidades e instituciones y las condiciones ambientales de los entornos.

El presente escrito tiene como propósito establecer una reflexión en torno al hábitat y la vulnerabilidad, a partir de sus desarrollos conceptuales y diferentes enfoques. Lo anterior con el fin de otorgar insumos a la discusión de estos conceptos de naturaleza polisémica, siempre abiertos a nuevas interpretaciones. En un principio se hablará del hábitat: la evolución, la complejidad y la significación del mismo, con el fin de establecer una mirada general que permita entenderlo como asunto dinámico, complejo y emergente de relaciones sistémicas; posteriormente, se realizará un desarrollo conceptual histórico de la vulnerabilidad (se puede hablar de su génesis), a través de la visión de diferentes escuelas de pensamiento, hasta revisar algunos modelos conceptuales que plantean a la vulnerabilidad en un escenario a-tempore complejo y cómo es construida a partir de la relación (sinergia) de diferentes factores.

\section{EL HÁBITAT, DESARROLLO DEL CONCEPTO DESDE LO INSTITUCIONAL}

Idear teorías y modelos (Rubio, 1993) que permitan conocer y entender de manera holística la lógica de la existencia humana, es un supuesto ideológico que perdió lugar en la sociedad moderna, la cual se ha acostumbrado a aplicar soluciones lineales en un mundo con relaciones cíclicas, buscando la unificación de criterios y llevando a la pérdida de la diversidad. Este periodo puede denominarse, según Vidal (2002), "el fin de las utopías" y el hábitat puede ser una de estas, que emerge como respuesta, según Fique (2008), a una nueva crisis acerca de cómo vive y cómo podría vivir la humanidad en las ciudades del planeta. 
La discusión que se ha forjado desde que el hábitat se introdujo en el escenario de la acción global, no parece tener conclusiones finales (brindando un escenario de nuevas oportunidades de creación). El impacto de estas discusiones sobre las condiciones de vida de los pobladores en sus contextos naturales y sociales, no ha llegado a cambios de realidades y paradigmas notables, sino que se ha quedado en artículos, conferencias, informes, tratados e intenciones por parte de las instituciones globales y locales.

En el contexto mundial, las Naciones Unidas han jugado un papel importante en la búsqueda de la significación del hábitat, desde el punto de vista conceptual y operacional. Los esfuerzos por esta caracterización empezaron en 1976 con la Conferencia de las Naciones Unidas sobre Asentamientos Humanos (Hábitat I), realizada en Vancouver, Canadá. En esta conferencia se emitió la primera declaración y los primeros compromisos en cuanto al hábitat, la cual determinó la necesidad de crear políticas, planes y programas nacionales e internacionales para los asentamientos; políticas públicas de derecho a la vivienda, el mejoramiento de la infraestructura y los servicios públicos; el acceso a la tierra y las políticas asociadas a ella; la participación pública, y la creación y coordinación de las instituciones que tengan que ver con la problemática.

A partir de esta declaración, firmada por los países asistentes, las Naciones Unidas crea el programa de las Naciones Unidas para los Asentamientos Humanos, UN-HABITAT, que tiene como objetivos, según UN-HABITAT (2009, pág. 1): "Facilitar el intercambio de información sobre vivienda y desarrollo sostenible de asentamientos humanos; colaborar en países a través de asesoría técnica para enfrentar los desafíos de la urbanización desordenada en las ciudades; promover vivienda digna para todos y todas". Además, se le encarga la tarea de organizar la segunda conferencia sobre asentamientos humanos.

La "Cumbre de las ciudades", como es conocida la conferencia Hábitat II, se llevó a cabo en Estambul en 1996. Para esta conferencia se parte del antecedente de Hábitat I y, se evalúan sus alcances en materia de resultados (formulación de políticas) acerca de los asentamientos. La evolución de la crisis humanitaria, político-institucional y ambiental en las ciudades, otorgó una "atmósfera" de impotencia a los países por causa de la complejidad del asunto y la necesidad de aplicar correctivos inmediatos y útiles a las realidades de las ciudades.

En este orden de ideas, "la Cumbre de las ciudades" trata de apartarse de la visión sectorizada y reduccionista del hábitat relacionado con la vivienda (a veces solo con la casa), y configura un programa de desarrollo como principio rector para la creación de asentamientos humanos sostenibles, que tengan en cuenta la relación con el ambiente, los derechos humanos, el desarrollo social y los derechos de la mujer; teniendo el programa una visión positivista de la urbanización, en el que la vivienda y servicios básicos, un empleo productivo, y un ambiente sano y seguro, son la directriz del programa.

El resultado de este programa es la Agenda Hábitat como guía de acción y monitoreo, según UN-HABITAT (2009) es un documento aprobado por los Estados Miembros, en el cual se comprometen a promover vivienda adecuada para todos y todas a la luz de asentamientos humanos sostenibles. La agenda hábitat reconoce en la contextualización del hábitat 
a la pobreza, la violencia, la calidad de vida, la familia, los derechos y deberes cívicos en la ciudad, los grupos vulnerables, los recursos financieros y la solidaridad de naciones "más ricas", y la salud en las ciudades.

En el plano institucional mundial, el hábitat sigue siendo relacionado con la idea de edificio aislado y solitario (de hecho allí se ha centrado la asignación de recursos), a pesar del esfuerzo de las conferencias de las Naciones Unidas para superar esa limitada concepción. En América Latina, las políticas de hábitat, responden a las necesidades de cada país por impulsar la construcción de vivienda. En el contexto local, en Colombia, se han materializado los postulados generales que dejaron las dos conferencias de Hábitat. Eso explica porqué, desde el punto de vista administrativo, es tan difícil ampliar el esquema hábitat/vivienda en el país, de hecho en la política de vivienda de Colombia, se incluye al hábitat no como contexto envolvente de la vivienda, sino como parte inherente a la construcción física de la misma. Es decir, el hábitat se circunscribe y nace de la acción de construir edificaciones (casas), a las que en la cotidianidad se les entiende como vivienda.

En la actualidad, en el país se tienen dos corrientes que abordan al hábitat de manera independiente (aunque en algunos casos se complementan) y aterrizan cada vez más este concepto. Estas dos maneras de atender la emergencia del hábitat desde la crisis humanitaria, social y legal son: la corriente administrativa e institucional del hábitat, en la que se encuentran los instrumentos de planificación que materializan los tratados firmados a la ONU; y la corriente académica, que trata de abordar de manera conceptual y contextual al hábitat desde una perspectiva sistémica e integral.

En Colombia se conocen dos ejercicios de planificación en términos del hábitat: los lineamientos de la política del hábitat de Cundinamarca, que tienen como objetivo, dotar a los municipios de instrumentos y herramientas que permitan mejorar su acción y lograr, bajo un marco común de principios y directrices, condiciones para enfrentar de manera coordinada la búsqueda de mejores alternativas de vida en materia habitacional y ambiental (Cundinamarca, 2004). Por su parte, desde el punto de vista administrativo, en la ciudad de Bogotá se creó la Secretaría del Hábitat, que tiene como misión garantizar la planeación, gestión, control, vigilancia, ordenamiento y desarrollo armónico de los asentamientos humanos de la ciudad en el aspecto habitacional (Bogotá, 2011).

En los dos instrumentos de planificación (y en las conferencias de las Naciones Unidas) mencionados anteriormente, se evidencia la concepción viviendista, la que se ha convertido en una estrategia más para la obtención de recursos de las políticas neoliberales, en la construcción de nuevos discursos desarrollistas, y en un mercado para los constructores (formales e informales) y urbanizadores, los cuales solo administran la pobreza del hábitat (en el contexto actual), y desde este panorama no aportan a la claridad del concepto de hábitat, lo que hace que sea susceptible de múltiples interpretaciones. 


\section{HÁBITAT MÁS QUE OBJETO MATERIAL, OBJETO DE ESTUDIO}

El discurso que las instituciones armaron para el hábitat, puede distar mucho de la realidad misma del concepto. Por lo general, la interpretación del hábitat se suscita a componentes físico-espaciales, dejando de lado un sinnúmero de elementos que lo constituyen y múltiples relaciones que lo definen y re-definen. Es aquí, donde se hace importante el uso de la complejidad para lograr un acercamiento real o al menos lo más acertado posible.

El término de hábitat empezó a ser utilizado por la ecología, según Chardon (2012) para referirse al espacio físico-natural donde se desenvuelve o desarrolla una especie animal o vegetal, pero según González (2002) poco después se fue aplicando dicha noción para designar los ámbitos o entornos donde el hombre habitaba. Es importante indicar y separar las nociones ecológicas y viviendistas del hábitat para entenderlo y abordarlo como objeto de conocimiento.

Se debe resaltar que el hábitat no es un objeto material (el discurso del desarrollo lo mimetiza así), y mucho menos se debe relacionar solamente con la huella física que el hombre deja al habitar un espacio. El hábitat visto desde la complejidad deja de ser estático y se recubre de relaciones dinámicas, lo cual permite que no se cosifique y se deje de apreciar como objeto aislado, describiendo en sí, una serie de procesos en permanente interacción-retroalimentación. Estos procesos van más allá de las técnicas de construcción, producción y ocupación, pues están orientadas a la génesis misma de la diversidad de realidades de quienes lo viven y lo construyen.

De este modo, las relaciones del sujeto con el hábitat (sujeto-sujeto) propician una multiplicidad de interpretaciones y significaciones, las cuales se pueden describir desde dos aristas: la primera permite, según Fique (2008), entender al hábitat como "un campo conceptual y no como simple objeto material"; la segunda, como un escenario donde se desarrollan historias de vida, una construcción social en la que interviene la cultura y los eventos que hacen posible la creación de hábitat.

\section{VISIÓN COMPLEJA DEL HÁBITAT}

El hábitat emerge de la articulación entre la naturaleza y la sociedad; cuando se habla de hábitat, no se hace alusión ni a la naturaleza, ni a la sociedad por separados, sino a la interrelación entre ambas. Esta comprensión de la realidad, según Ossa (1981), nace desde las interrelaciones de sus elementos constitutivos, que en el caso del hábitat, según Fernández (2000), se pueden reconocer al igual que el ambiente como sistemas.

Un importante punto de partida en la conceptualización del hábitat, es el hecho de comprenderlo como sistema, lo que significa identificar un conjunto de criterios generales a través de los cuales podemos establecerlo como tal. Capra (1998, pág. 172) propone tres dimensiones conceptuales, a saber: 
i) el patrón de organización, que es la configuración de las relaciones entre sus componentes, que determina las características esenciales del sistema; ii) la estructura, que es la corporación física de un patrón de organización; y iii) el proceso, que se ocupa del patrón de organización del sistema. Siendo éste, el criterio que constituye el vínculo entre patrón y estructura.

El patrón de organización es el hábitat y las estructuras de este son, según Fique (2008), la sociedad que tiene acciones sobre el entorno físico y la naturaleza que re-acciona a la construcción del medio. El tercer componente que permite la interrelación de las estructuras, es el proceso, conocido también como cultura, la cual según Ángel Maya (1996) es un mecanismo para-biológico de adaptación al medio, y que complementa Gómez (2000 citado por Fique, 2008) como los cambios permanentes en la elaboración de las estructuras simbólicas que se involucran a la estructura social y a las estructuras físicas, un encuentro dialéctico, para transformar en este caso al hábitat (Figura 1).

La relación de la arquitectura y las ciencias ambientales con el hábitat, es abordada a partir de los trípticos que conceptualizan estas disciplinas. Desde la arquitectura: vida, lugar y técnica se plantean, según Fique (2008), como los referentes básicos del quehacer arquitectónico, que coincide con el análisis ambiental propuesto por Santos (2000, pág. 91), donde la técnica (mediada por la cultura) es la principal forma de relación entre hombre y naturaleza, constituyendo un conjunto de medios instrumentales y sociales, con los cuales el hombre realiza su vida, produce y, al mismo tiempo, crea espacio.

Evidenciando la correspondencia que entre arquitectura, ambiente y hábitat existe, Fique (2008) propone un modelo conceptual que articula los patrones, estructuras y procesos, llegando a la conclusión de que estos tres conceptos poseen similares estructuras y estas se articulan mediante los mismos procesos. La única diferencia (estructura y proceso), es la escala de acción y conocimiento, que complementa a los tres conceptos y los integra, relacionándolos desde la técnica.

Con base en lo anterior, se puede plantear un esquema de comprensión (Fique, 2008) (Figura 1) donde el hábitat, como asunto complejo, surge de nuevo en la relación de las estructuras mencionadas anteriormente, sin embargo, esta vez se muestran los procesos de producción simbólica y material, reconocidos como técnicas y prácticas del habitar. 
Figura 1. Modelo conceptual de hábitat

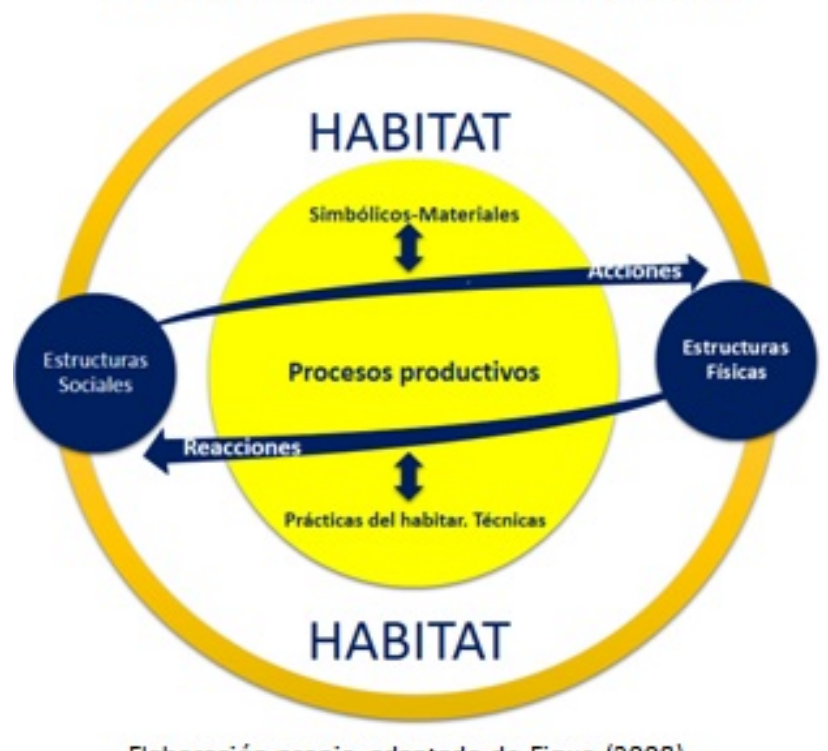

Elaboración propia, adaptado de Fique (2008)

El modelo conceptual del hábitat (Figura 1) hace énfasis en los procesos sobre los cuales las estructuras sociales se basan para apropiar, modificar y construir el medio, como un instrumento para adoptarlo y significarlo. Un proceso productivo del hábitat, según Fique (2008, pág. 117), "es dinámico -en movimiento cambiante- y complejo -pleno de variables e interrelaciones- de toma de decisiones, en el cual cada agente actúa desarrollando estrategias y haciendo uso de técnicas que buscan atender a sus valores y satisfacer sus intereses".

Desde esta perspectiva planteada, los procesos se convierten en objetos de conocimiento y no en objetos materiales, así al hábitat se le puede abordar y entender como un marco contextual de estructuras y procesos, que contiene amenazas y por lo tanto vulnerabilidades. Escenarios en los cuales se desarrolla el hombre y que se encuentran relacionados con las prácticas del habitar.

\section{LA ACCIÓN DEL HÁBITAT: HABITAR}

El habitar se refiere, según Heidegger (1994, pág. 2), a la manera según la cual "los hombres somos y estamos en la tierra. El rasgo fundamental del habitar es custodiar y velar en lo libre", desde las creencias, identidades y objetos; llegando a la conclusión de que el habitar es un residir junto a las cosas, en otras palabras donde el hombre está, es, tiene e interpreta su manera de vivir.

Las formas de habitar de las personas y la sociedad construyen al hábitat y las condiciones que hacen que sea vulnerable, siendo este un asunto dinámico en continuo movimiento, en perpetua (a escala humana) construcción, según Echavarría (2004, pág. 31): 
[...] nunca se deja de habitar los lugares ni siempre se los habita de igual forma. Los habitantes que ejercen tal acción nunca son los mismos, pues, aunque muchas veces las personas en sí mismas no cambien, el curso de tiempo implícito en toda acción, lleva a que ellos, en sí mismos cambien en cada momento, variando los propios sentidos de su accionar, y por ende los sentidos del lugar que se construye.

La acción de habitar, va más allá que el ocupar un lugar, según UNHABITAT (2012) trasciende de la localización física en un territorio a su apropiación y adaptación -mediada por la cultura-. Por tal motivo, se puede afirmar, según Giraldo (2003, pág. 43), Que "el ser humano se localiza no solo en términos físicos y biológicos, sino en una multidimensionalidad, natural y social, ordenada y simultánea, propia de cada sociedad, de cada territorio". Desde este punto de vista, el hábitat se puede entender, como el sitio de permanencia, el sitio donde el hombre reside, en otras palabras, habita, pero en el que es necesario que sea enriquecido por la presencia de muchos individuos - no solo humanos, sino también animales domésticos, por ejemplo-, que le den sentido y lo habiten.

Reconocer el hábitat desde el habitar, según Echavarría (2011, pág. 1), "implica pensarlo desde la diversidad de sujetos individuales y colectivos, con sus sentidos existenciales, móviles e intereses, necesidades, vacíos, movimientos, recorridos, expresiones, usos y materializaciones". Es así que el habitar, la acción continua del hábitat, se traduce en la transformación del espacio por el hombre y en los hombres, y la conversión del hombre por el espacio en los espacios, sobrellevando esto a una construcción dialéctica y compleja, en donde el equilibrio de esta construcción es móvil, no estático.

El habitar se configura a distintas escalas, que pueden ir desde lo micro (la vivienda, la habitación), hasta lo macro (el país, el continente). Estas escalas pueden ser conectivas como los caminos, calles y aceras, o pueden ser construcciones sociales como las viviendas, comunas y barrios, teniendo en común que ambas describen las formas de habitar de sus habitantes, los cuales construyen hábitat desde su interior y lo exteriorizan en el contorno, en lo público, por eso, Echavarría \& Rincón (2000, pág. 43) afirman que "el habitar construye su hábitat más allá de la esfera de las relaciones domésticas en su hogar, [de allí que no se limite a las esferas micros o macros, sino que esté marcado] por los sentidos de la vida urbana".

De acuerdo a lo anterior, el habitar se puede, según Echavarría (2011, pág. $5)$ :

[...] reconocer como acontecer y hecho, referido a aquellas tramas de vida que auto-producen grupos humanos particulares en relación con los otros, en concordancia con las valoraciones, idearios, imaginarios, deseos, circunstancias y posibilidades, que se pueden expresar, o no, en materialidades, acciones y prácticas cotidianas, configurando entornos habitados signados por lógicas propias: espaciales, culturales, económicas, sociales y políticas. 


\section{SIGNIFICACIÓN DEL HÁBITAT Y SU RELACIÓN CON LA VULNERABILIDAD}

Teniendo en cuenta las reflexiones anteriores, se puede argumentar que el hábitat es una construcción humana, que contiene relaciones de espacio (Bordieu, 1997) y tiempo (desde las dimensiones social y natural), desde las perspectivas del estar, el ser y el tener. Según González (2002), haciendo alusión al origen francés de la palabra, no es exactamente el lugar donde se vive, sino el lugar donde se tiene. Siendo los bienes inmuebles, las tierras y la "habitación" lo que obliga a "habitar". En este contexto, el concepto pasa de ser soporte a contenedor de una sociedad, que marca, posee y transforma la base (soporte) con motivaciones, memorias e imaginarios. Donde el hombre apropia y significa de forma continua el espacio, volviéndolo territorio.

El territorio se puede entender, según Chardon (2012, pág. 7), como "un espacio con actores, dueños, defensores y dolientes con sentido de pertenencia hacia esta unidad espacial en la cual se reconocen, son reconocidos porque participan de su construcción y desarrollo". En tal medida, generar sentido de pertenencia e interdependencia a un lugar, es territorializar, y es importante reconocerlo ya que esta condición hace al hábitat único, endémico y crea multitud de hábitats (casi igual al número de personas que existen) que se yuxtaponen, interpolan y co-existen.

Por su parte, Leff (1998, pág. 280), reconoce al hábitat como la:

[...] espacialidad de una sociedad y de una civilización, donde se constituyen los sujetos sociales que diseñan el espacio geográfico apropiándoselo, habitándolo con sus significaciones y prácticas, con sus sentidos y sensibilidades, con sus gustos y goces, [agrega además, el hábitat] es el espacio donde se desarrollan las actividades productivas, culturales, estéticas y afectivas del hombre. Es el medio donde los seres vivos evolucionan y complejizan su existir, donde el organismo social despliega sus potencialidades, es espacio donde define su territorialidad.

Esta territorialidad es creada a partir del hábitat, se conforma por medio de las prácticas y actividades que modifican y crean el entorno, al tiempo que genera soporte de vida para los hombres y, según Leff (2004), es el lugar significado por experiencias subjetivas, de vivencias construidas con la materia de la vida. El cual, al ser subjetivo y propio de las ciencias blandas, es susceptible de ser abordado por parte del pensamiento complejo (Morin, 1995; Noguera, 2006).

El pensamiento complejo permite la edificación de nuevos conceptos, métodos y teorías que hagan visibles la naturaleza compleja y dinámica del hábitat humano sumido en un contexto biodiverso (desde lo natural y lo cultural) vulnerable. Las personas construyen sus hábitats y los viven con las diversas vulnerabilidades, carencias y desequilibrios, afectados por las dinámicas económicas, sociales, culturales, físico-espaciales y ambientales, reflejado en las adaptaciones de las poblaciones en la construcción de nuevas formas de habitar.

El hábitat comprende el contexto en el cual los individuos y sus comunidades establecen redes de relaciones cíclicas y continuas, 
enmarcadas en diversos procesos que permiten la configuración gradual en el espacio-tiempo de vulnerabilidades asociadas a amenazas. Lo anterior, permite establecer un encuentro de interrelaciones cíclicas y complejas en el que las poblaciones intervienen, modifican y ocupan los espacios (físiconaturales) para construir hábitat, construyendo y potenciando a su vez (en algunas ocasiones) escenarios vulnerables.

La anterior reflexión permite comprender, según Chardon (2004, pág. 2), que "el hábitat contiene las amenazas naturales, las cuales, en ningún momento se pueden considerar como un elemento externo, hacen plenamente parte del hábitat, son producto de él, es decir del sistema de vida", y es allí, donde se hace imposible separar al hábitat de la vulnerabilidad, pues en la construcción de un hábitat siempre van a existir factores que potencien la vulnerabilidad, y en el continuo ciclo de superar esas condiciones vulnerables (para mejorar la calidad de vida) se modificará el hábitat.

\section{LA VULNERABILIDAD COMO CUESTIÓN COMPLEJA}

Se habla de que la vulnerabilidad es una cuestión compleja debido al concurso de diversas profesiones e instituciones, a la confluencia de factores multidisciplinarios que la configuran y a la heterogeneidad de los sistemas físicos-naturales en los que se gesta. La vulnerabilidad puede ser entendida como una problemática -compleja- no resuelta del desarrollo (Escobar, 2007), por eso es evidente la dificultad que esta reviste en los países "tercermundistas", donde el esquema de modelo de desarrollo adoptado por la sociedad (del país) es el principal factor de las situaciones vulnerables en los contextos más locales.

A continuación, se exponen los distintos enfoques que dieron origen al concepto de la vulnerabilidad, hasta el desarrollo de teorías y modelos conceptuales que permiten la construcción de métodos de análisis y la comprensión de la vulnerabilidad en un contexto histórico, el cual-para que tenga validez- debe ser abordado desde la escala del hábitat.

\section{EVOLUCIÓN DEL CONCEPTO DE VULNERABILIDAD}

En la actualidad no se encuentra un consenso en el concepto de vulnerabilidad (lo cual no sería conveniente); aún se producen teorías y metodologías de amplia aceptación en el contexto académico. Los conceptos reflejan posiciones y enfoques que han desarrollado todo un discurso, influenciado y enriquecido, claro está, desde las diferentes disciplinas, llevando a que se incluyan aportes desde las ciencias naturales, aplicadas y sociales, que han derivado en la construcción e inclusión de modelos de vulnerabilidad más complejos y menos reduccionistas.

\section{a) Enfoque de las ciencias naturales}

El primer periodo de investigaciones acerca de la problemática del riesgo, la amenaza y la vulnerabilidad, se realizó desde los aportes de las ciencias naturales. En este enfoque se consideraba a los desastres como fenómenos extremos producto de la naturaleza, según Maskrey (1998, pág. 
12) "se postulaba que un terremoto, erupción volcánica, huracán u otro evento extremo era de por sí un desastre", y la investigación de los desastres se abordó desde disciplinas como la geología, la meteorología, la hidrología, la sismología y la física, entre otras, con el fin de predecir el comportamiento de los fenómenos naturales en las escalas del espaciotiempo, con variables a describir como la distribución espacial, la periodicidad y la magnitud de las amenazas.

El desarrollo de este enfoque potenció un avance tecnológico en el estudio de los eventos naturales (Quarantelli, 1987), a tal punto que ha permitido, según Cardona (2003, pág. 5), "la instrumentación de fenómenos naturales mediante sensores, incluso en tiempo real, que permiten dar alertas o avisos anticipados de sucesos intensos". Esto generó la conformación de sistemas de alerta temprana y de seguimiento a fenómenos naturales, como el caso de volcanes, huracanes, tsunamis, entre otros, los cuales han posibilitado salvar algunas vidas, ya sea porque el grupo humano alcance a organizarse para enfrentar el fenómeno, o simplemente, se hagan los preparativos para la evacuación del área de influencia de un suceso severo.

El desarrollo tecnológico favoreció específicamente el conocimiento de los fenómenos naturales, pero generó, según Hewitt (1983), Maskrey (1998) y Cardona (2003), una confusión entre el riesgo y la amenaza. Si bien el aporte de las ciencias naturales a la estimación del riesgo es necesario, no se puede reducir la problemática excluyendo a la vulnerabilidad y simplificando el riesgo a un potencial de pérdidas, en caso del suceso de un fenómeno natural futuro.

Por otra parte, este enfoque ha favorecido la creencia de que los eventos extremos, son atribuibles a hechos del destino, causas sobrenaturales o consecuencias divinas, debido a que algunos eventos, como es el caso de los terremotos, son difíciles de predecir ( $\mathrm{y}$ entra el saber popular a explicarlos). Por este motivo, algunas poblaciones asimilan estos eventos a "la acción de dios". Por ejemplo, el terremoto de Haití en el año 2010 es percibido como consecuencia de un castigo por las prácticas de "magia negra" propias de la cultura de la isla, dejando a un lado, la discusión de la vulnerabilidad asociada a la pobreza extrema de gran parte de la población del país caribeño.

Desde el contexto institucional se ha aprovechado la confusión generada por el enfoque de las ciencias naturales, en la descripción de las causas y consecuencias de los fenómenos naturales, para evadir responsabilidades que tienen los mandatarios, las instituciones y la sociedad en general. Un ejemplo que hace gráfica la idea anterior, puede ser la explicación del exdirector del ente descentralizado de cultura de Manizales, ante la crisis del agua y la tragedia del barrio Cervantes en el año 2011, donde establece que la ciudad estaba "maldita" y que había que realizar rezos y riegos en la plaza principal de la ciudad2 . Lo anterior representa una manifestación cultural, un rito propio de la cultura cafetera, pero eclipsa la discusión de fondo, es decir, la responsabilidad de las autoridades y de la comunidad en estas dos situaciones que afectaron a la ciudad.

Por último, el gran logro de la investigación que se realizó bajo este enfoque, fue la declaración del DIRDN, por las Naciones Unidas a finales de la década de los ochenta, donde se definieron los campos de acción, según Maskrey (1998, pág. 13), "para mejorar el conocimiento asociado al riesgo y difundir información sobre las amenazas naturales, coherentes con 
el enfoque de las ciencias naturales en general". Evidenciando, en la terminología asociada a la declaración, la categorización de los desastres según el tipo de amenazas.

\section{b) Enfoque de las ciencias aplicadas}

El enfoque de las ciencias aplicadas ofreció un cambio de paradigma en el estudio de las amenazas. Se empieza a debatir en el escenario del riesgo la inclusión de la vulnerabilidad y la asociación que pueda tener con las amenazas, y el tema de la transferencia del riesgo, entre otros, enriquecidos por la confrontación suscitada de las corrientes de pensamiento de los enfoques natural y social.

Este enfoque se caracterizó por el interés de estudiar el impacto de los eventos en su relación con las amenazas, según Maskrey (1998, pág. 13), "en el espacio, en sistemas constructivos, en las morfologías urbanas, en redes de infraestructura, etc.". El concepto que hacía iguales a riesgos y amenazas, evoluciona hasta determinar la relación de los desastres con los impactos producidos en contextos sociales, económicos y ambientales.

Varios autores hacen evidente este cambio en el paradigma, por ejemplo Cuny (1983) considera a los eventos extremos como los catalizadores que transforman una condición vulnerable en desastre, incluyendo a la vulnerabilidad en el contexto del desastre; por su parte, Cardona (1992, pág. 46) reafirma esta posición definiendo a los desastres "como un evento no esperado, que causa alteraciones intensas a elementos expuestos; por ejemplo, la muerte, daños a la infraestructura o cambios ambientales".

Surge el concepto de la vulnerabilidad resaltando que los eventos y las amenazas no son homogéneos, por el contrario, son discontinuos en el espacio-tiempo y son medidas del desorden, es decir de la entropía. Según Romero \& Maskrey (1993, pág. 16), "el riesgo se empezó a definir en función tanto de la amenaza como de la vulnerabilidad". Siendo a partir de este momento, importante estudiar las propiedades físicas e intrínsecas de un sistema expuesto a una amenaza, considerándose este aspecto como el aporte de las ingenierías y las ciencias duras.

La producción de mapas que zonificaban las amenazas en un espacio geográfico y las herramientas informáticas como los sistemas de información geográfica -SIG-, facilitaron los análisis y las predicciones en el comportamiento de los sistemas y las personas ante un escenario de riesgo, en las escalas de tiempo de los desastres (antes, durante y después), permitiendo la acumulación de datos estadísticos, la generación de matrices y modelos de simulación probabilísticos más complejos. Para lo cual ha sido necesario el concurso de geógrafos, geólogos, hidrólogos, gestores ambientales, economistas, planificadores, estadísticos, etc.

Este enfoque del riesgo es determinado a partir de la cuantía de los daños que puedan sufrir los sistemas afectados por los eventos catastróficos y la modelación de la probabilidad de la amenaza. Esta posibilidad de abordaje de la problemática del riesgo, la amenaza y la vulnerabilidad, según Cardona (2003, pág. 6), "favorece que los resultados obtenidos puedan traducirse en pérdidas potenciales y puedan aplicarse, bajo el concepto de la relación costo/beneficio en la elaboración de códigos de construcción, normas de seguridad y planeamiento urbano", permitiendo que el riesgo se 
convierta en una variable objetiva y medible, haciendo que sea un poco más fácil su cuantificación.

El enfoque de las ciencias aplicadas, reconoce la existencia de la responsabilidad de comunidades e instituciones, en las pérdidas que se puedan presentar ante un evento. Establece que la sociedad civil y el gobierno, son competentes en la implementación de las medidas necesarias para la reducción de los riesgos, dejando a un lado, la idea de ingobernabilidad que se tenía (y se mantiene hoy en día) sobre los riesgos.

\section{c) Enfoque de las ciencias sociales}

El enfoque de las ciencias aplicadas, produjo cambios en la percepción que tenían los científicos en cuanto al riesgo, mostrando un avance importante en la intención de abordar esta problemática integralmente. A este cambio, se le sumó el énfasis producto de la intervención de los científicos sociales, que empezaron a cuestionar los supuestos establecidos del anterior enfoque con respecto al tema de la vulnerabilidad.

El desarrollo de una teoría social de los desastres en Estados Unidos (en los años 20 del siglo $X X$ ), generó una serie de conceptualizaciones y un enfoque social, que empieza a centrarse en las percepciones de las poblaciones ante los desastres. De esta manera, cambia la dirección de las investigaciones que centraban su estudio en la reacción o respuesta de las poblaciones ante el desastre (en el momento del desastre), hacia la comprensión del riesgo como objeto de estudio y en el marco de las políticas púbicas de gestión del riesgo (Quarantelli, 2001; Lungo, 2002).

El aporte realizado desde la geografía y los saberes ambientales al enfoque de las ciencias sociales, ayudó a establecer que un desastre no es lo mismo que un evento natural y, según Cardona (2003, pág. 6), "sus aportes en relación con la necesidad de considerar la capacidad de reacción, o de ajuste de una comunidad, ante la acción de eventos naturales o tecnológicos fue la base conceptual del concepto de vulnerabilidad".

El enfoque de las ciencias sociales ayudó a determinar que los desastres, además de ser causados por fenómenos físicos de la naturaleza, eran causados, también, por las sociedades expuestas a las amenazas de un sitio. Interesándose así los investigadores sociales, en el conocimiento de la percepción individual y colectiva (en el contexto social) de las amenazas, relacionado con las decisiones tomadas por las comunidades e instituciones, influenciadas por las realidades económicas, culturales y ambientales de sus hábitats, en otras palabras, la vulnerabilidad de las poblaciones expuestas.

Se plantea que la vulnerabilidad tiene un carácter social y que no se puede circunscribir solamente a la cuantificación de los daños físicos. Según Westgate \& O’keefe (1976, pág. 35), se postula que "la vulnerabilidad no se puede definir o medir sin hacer referencia a la capacidad de la población de absorber, responder y recuperarse del impacto del suceso". Por tal motivo, un mismo evento puede afectar de diversas maneras a varias poblaciones, por ejemplo, los países pobres se ven más afectados ante un desastre debido a sus frágiles condiciones sociales, a la pobreza y a la transferencia del riesgo, confirmando así un postulado de Susman et al. (1984, pág. 266), que establece a la vulnerabilidad como "el grado en que las diferentes clases sociales están diferencialmente en riesgo". 
La vulnerabilidad se establece de acuerdo a las condiciones sociales, económicas, culturales, ambientales y políticas de la población. Haciendo que esta sea diferente en cada zona, en cada tiempo y para cada persona. Desde esta perspectiva, es posible afirmar que las condiciones históricas de un grupo humano como las políticas, guerras, conflictos, modelos económicos y de explotación; la historia ambiental del territorio representado por las transformaciones sociales de los entornos naturales, el historial geológico y meteorológico; y las características culturales de las poblaciones, manifestadas en los mitos, ritos, educación, creencias e idiosincrasia, hacen que las comunidades pobres sean más vulnerables a las pérdidas de bienes materiales e inmateriales en los desastres, producto del alto grado de deterioro ambiental del entorno en donde habitan.

Este enfoque habla de una configuración histórica de la vulnerabilidad, es decir, esta no aparece en el momento del impacto del evento, sino que nace de la sinergia histórica de los diferentes factores de vulnerabilidad (una construcción histórica), se manifiesta en el evento y continúa visible en el futuro. Lo anterior permite concluir que, según Maskrey (1998, pág. 15), "la vulnerabilidad es una condición socialmente producida". En relación con lo anterior, Lavell (1992, pág. 79) precisa a la vulnerabilidad como "periodos de crisis en el marco de los procesos sociales preexistentes en una sociedad".

En el contexto latinoamericano, la Red de Estudios Sociales en Prevención de Desastres en América Latina -La RED-, según Cardona (2001, pág. 16), "ha planteado que la vulnerabilidad se configura socialmente y es el resultado de procesos económicos, sociales y políticos", por tal motivo, al momento de realizar un estudio de la vulnerabilidad se hace necesario comprender factores sociales como la inexistencia de saneamiento básico en las comunidades, el déficit de viviendas (en calidad y cantidad), los ingresos familiares y el empleo, la violencia intrafamiliar y territorial, la corrupción de las instituciones, la discriminación por raza y creencias políticas, el analfabetismo y la degradación ambiental, entre otros.

El enfoque de La RED en la conceptualización y el diseño de metodologías, ha contribuido en la comprensión de la complejidad del riesgo. El enfoque latinoamericano a la problemática del riesgo ha brindado otra perspectiva, que visibiliza a las personas que se ven afectadas por "catástrofes", centrándose en el análisis de las causas estructurales y en las consecuencias sociales y ambientales (Lampis, 2010), desde la crítica constructiva de los modelos económicos globales, los estilos de vida de las poblaciones y las decisiones político-administrativas.

Comparar los tres enfoques con los que el tema de la amenaza, la vulnerabilidad y el riesgo ha sido abordado, resulta una tarea interesante en la construcción histórica de los conceptos; si la intención de este análisis comparativo, permite establecer las similitudes y encontrar en las diferencias las posibilidades de acercamiento y re-construcción de los conceptos, es decir, se debe entender que el desarrollo de cada enfoque no es excluyente de los demás, por el contrario, son mutuamente complementarios, pues describen una sola realidad, el riesgo. 


\section{VULNERABILIDAD, CONCEPTO DINÁMICO EN CONSTRUCCIÓN}

Se entiende que la vulnerabilidad es un concepto en construcción, no es una situación en sí, sino que se puede entender y abordar como la relación dinámica de múltiples y variadas situaciones, actores (entiéndase también agentes), decisiones, condiciones, políticas, entornos y disciplinas, entre otras. Siempre está asociada a amenazas naturales, antrópicas y socionaturales, y es subsistema de un sistema de mayor jerarquía, el riesgo.

La vulnerabilidad no es un escenario estático, por el contrario, es un sistema dinámico que cambia constantemente en el espacio-tiempo de acuerdo con las amenazas que la originan y a los hábitat en los que se presenta; a esto se le debe sumar que pertenece al campo de la incertidumbre y el azar, lo que la convierte en relativa y propia de cada lugar o comunidad, y evoluciona, según Chardon (2008, pág. 10):

[...] cualitativa como cuantitativamente: 1- en el tiempo, para una comunidad expuesta a una misma amenaza; 2en el espacio en un momento determinado, para varias comunidades expuestas a una misma amenaza; y 3- según el tipo de amenaza, puesto que las fragilidades/debilidades varían en función del tipo de peligro.

Lo anterior indica que la vulnerabilidad no pertenece a un tiempo específico, asociado comúnmente al momento del impacto de un desastre, sino más bien, evoluciona gradualmente en el tiempo, implicando un antes, un durante y un después del desastre.

Como asunto complejo, la vulnerabilidad debe ser entendida como un sistema en el que los procesos cíclicos (no lineales) que subyacen del mismo afectan a los factores estructurales y no estructurales de vulnerabilidad, en una comunidad o grupo humano en particular (Maskrey, 1989; Medina \& Romero, 1992). Estos factores, según Chardon (2008, pág. 9), "pertenecen a campos físico-naturales, ecológicos, sociales, económicos, físico-espaciales, territoriales, tecnológicos, culturales, educativos, funcionales, político-institucionales y administrativos o coyunturales principalmente", y se hacen particulares a un territorio y grupo humano debido a la sinergia generada por sus interrelaciones.

Por otro lado, si bien el presente escrito no hace especial énfasis en los conceptos de amenaza y riesgo, estos se han mencionado $-\mathrm{y}$ se mencionarán- con frecuencia, lo que hace importante definirlos con el fin de no simplificarlos y reducirlos (permitiendo realizar un paréntesis en el tema de vulnerabilidad). La amenaza, es un fenómeno que puede representar peligro para un grupo humano, según Cardona (2003, pág. 14), "es la posibilidad de ocurrencia de un evento potencialmente desastroso durante cierto periodo de tiempo en un sitio dado". En el contexto práctico es posible evidenciar varios tipos de amenazas, por ejemplo, naturales, socionaturales, antrópicas, tecnológicas, biológicas, etc.

La amenaza como factor externo a las comunidades, hace referencia a los eventos que puedan impactar los bienes materiales e inmateriales del hombre, incluso la vida misma. Según Foschiatti (2012), tiene tres componentes: energía potencial, susceptibilidad y detonador, los cuales se encuentran relacionados (Figura 2) y funcionan como un sistema, es decir 
son diferentes de acuerdo a la sinergia que presentan, el grado y la forma de la relación de sus componentes.

Figura 2. Componentes de la amenaza

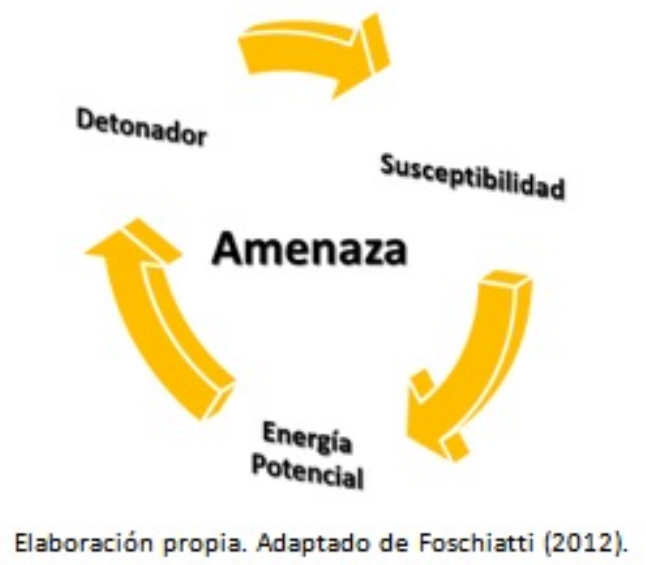

El concepto de amenaza se puede interpretar como un peligro latente, el cual asociado con la vulnerabilidad, crea el riesgo. Este último, según Chardon (2008, pág. 10), "resulta del cruce probable en el espacio como en el tiempo entre una amenaza de magnitud determinada y un elemento relativamente vulnerable a ella". De esta manera, el riesgo puede ser considerado como el potencial de pérdidas que le pueda ocurrir a un sistema expuesto.

La amenaza existe en la medida en que exista la vulnerabilidad, son dependientes, concomitantes y complementarias, según Cardona (2003, pág. 9), "no se puede ser vulnerable si no se está amenazado y no existe una condición de amenaza para un elemento, si no se está expuesto y es vulnerable a la acción potencial que representa dicha amenaza". Por tal motivo, el riesgo es relativo, se mueve en el escenario de las probabilidades, es dinámico y depende de la relación de dos factores complejos.

\section{MODELOS CONCEPTUALES DE VULNERABILIDAD}

En los apartados anteriores, se realizó un recorrido histórico que mostraba la génesis del concepto de vulnerabilidad. Si bien, se concluye que la vulnerabilidad es una construcción social, en la conceptualización se podría decir que es una construcción colectiva, que aún no encuentra consenso, que aún no llega a su fin. Para efectos de esta construcción, se mencionarán los principios de algunos modelos conceptuales de la vulnerabilidad que permiten aterrizar una mirada integral de este concepto.

A finales de los años 80 , surgen modelos de vulnerabilidad que buscan el análisis integral de las situaciones que la generan y potencian, haciendo uso de la interdisciplina y la articulación de los contextos sociales-naturalesespaciales. Uno de los primeros estudios fue desarrollado por Anderson \& Woodrow (1989), en el que se identifican tres clases de vulnerabilidad: físico-material, social-organizacional y motivacional-actitudinal (Figura 3). 
Ulteriormente, aparecen los estudios de Wilches-Chaux (1989, pág. 11), donde se considera que "la vulnerabilidad no es una situación en sí, sino que es la relación de varios tipos de vulnerabilidad, clasificándola en física (locacional), económica, social, política, técnica, ideológica, cultural, educativa, ecológica e institucional" (Figura 3). Este enfoque en particular, otorga una mirada holística a la vulnerabilidad y permite ampliar el análisis, que obedece a la complejidad del concepto, aunque se debe reconocer que no se hace mención a los factores de la vulnerabilidad.

Estudios como el de Ratick (1994), determinan que la vulnerabilidad depende de elementos como la exposición, la resistencia, la resiliencia, la recuperación, el aprendizaje y la adaptación a los desastres (en los tiempos pasado, presente y futuro). Estos elementos se presentan en función de las características (internas y externas) propias de una comunidad (Figura 3).

Así mismo, algunos autores como Blaikie et al. (1996, pág. 218) consideran "la vulnerabilidad como la evolución de un proceso", usando el término de "progresión de la vulnerabilidad" (Figura 3). Este modelo de presiónliberación tiene tres niveles que conectan el desastre con procesos relacionados en los contextos políticos y económicos. El primer nivel, son las Causas de Fondo que reflejan la distribución del poder en una sociedad, relacionadas con el acceso al poder, estructuras y recursos, y otros elementos del orden ideológico. Posteriormente, en este modelo, se encuentra un nivel de segunda categoría conocido como las Presiones Dinámicas (falta de instituciones e inversiones locales, libertad de prensa y ética en la vida pública), el cual canaliza las causas de fondo hacia las Condiciones Particulares de Inseguridad (tercer nivel manifestado por: fragilidad en ambiente-físico, economía, sociedad vulnerable y acciones públicas), siendo este último nivel, la forma específica de la vulnerabilidad asociada a una amenaza en el espacio-tiempo.

Por otra parte, Cardona (2001) propone la existencia de factores que originan la vulnerabilidad: la fragilidad física o exposición, la fragilidad social y la falta de resiliencia (Figura 3). El primer factor, relaciona las condiciones del área de influencia de los fenómenos peligrosos y su falta de resistencia física ante los mismos. El segundo factor denota las condiciones propias del grupo humano, y el tercer factor expresa la incapacidad de respuesta y reposición de las poblaciones afectadas. Una posición similar es expuesta por Turner et al. (2003), estableciendo que el marco de la vulnerabilidad se compone de tres elementos: la exposición de individuos, hogares, estados y ecosistemas; la sensibilidad de las condiciones humanas (capital social y humano) y las condiciones ambientales; por último, la resiliencia que muestra la articulación de la respuesta desde las situaciones de la toma de decisiones (políticas actuales), el impacto de los desastres y el ajuste de las poblaciones (nuevas políticas).

Por su lado, Foschiatti (2012, pág. 8) propone que "la vulnerabilidad depende del grado de exposición, de la protección, la reacción inmediata, la recuperación básica y la reconstrucción. El segundo y el tercero conforman la homeostasis y los dos últimos la resiliencia y ambas constituyen la resistencia" (Figura 3). Lo anterior, con el fin de determinar un campo de acción metodológico en la intervención sobre la vulnerabilidad. 


\section{Figura 3. Modelos conceptuales vulnerabilidad}

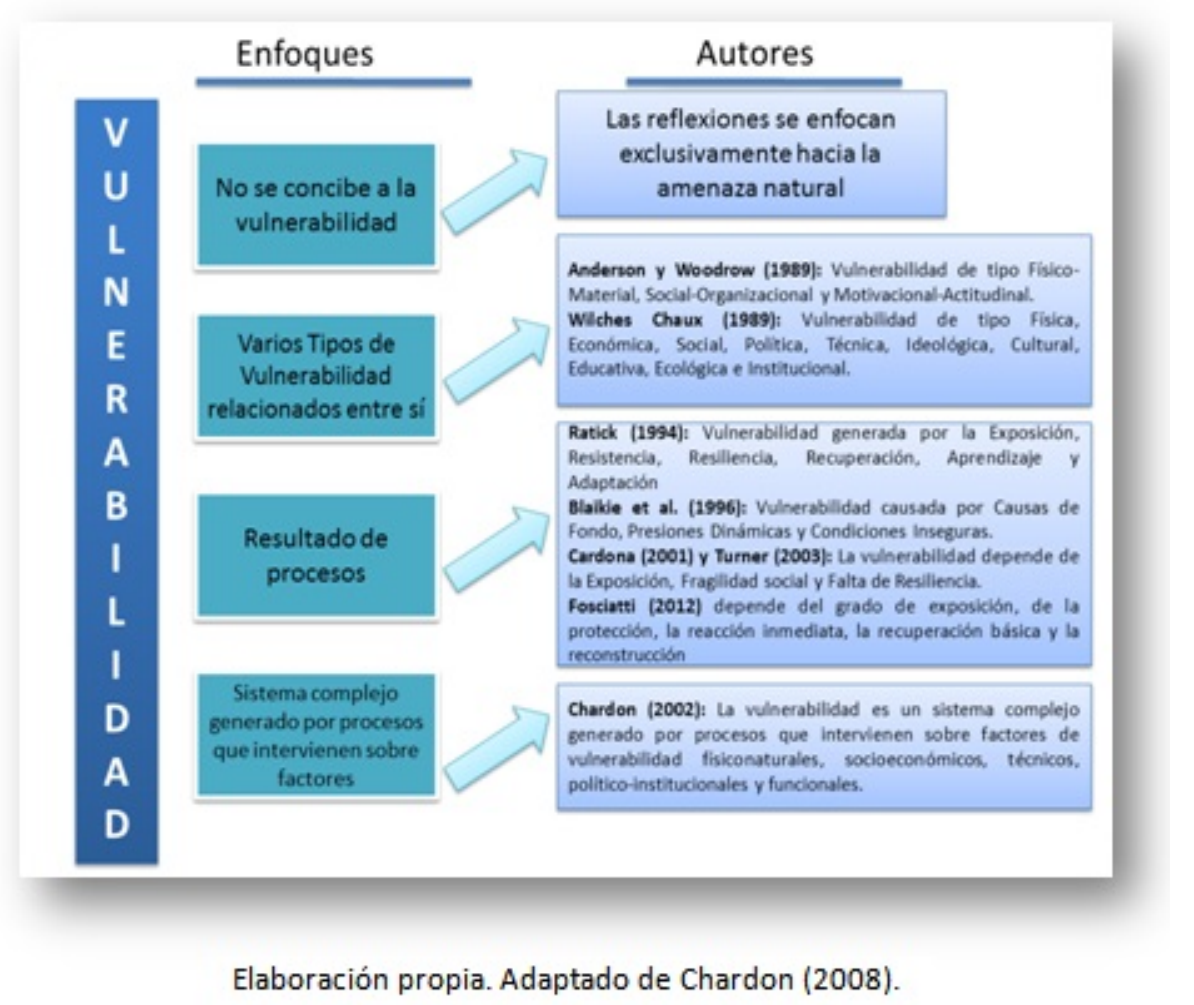

A su vez, Chardon (2008) propone desde una visión integral, la unicidad de la vulnerabilidad (una sola vulnerabilidad), estableciendo que el concepto se expresa en la relación de diferentes factores interrelacionados y asociados a procesos macro. Dichos factores se clasifican en físico-naturales, socioeconómicos, técnicos, político-institucionales y funcionales.

Los factores físico-naturales de la vulnerabilidad hacen referencia a la composición geo-estructural donde se basan las poblaciones, así como los fenómenos naturales que por cuestiones de ubicación y la acción de las poblaciones en el espacio se presenten (ejemplo zonas volcánicas, de ladera o de inundación). A su vez, los factores socio-económicos están relacionados con aspectos como: la tipología socio-económica de los barrios, el empleo, los ingresos económicos, la educación, las organizaciones sociales, los equipamientos colectivos, la atención a los desastres, el saneamiento básico, la infraestructura física de acceso y circulación que tienen los centros poblados.

Los factores políticos-institucionales, según Chardon (2008) corresponden a los elementos más determinantes de la generación cualitativa y cuantitativa de una situación de vulnerabilidad. Están representados por las políticas públicas, las características de las instituciones (y de su personal técnico) y la articulación territorial en la gestión del riesgo; la característica fundamental de este factor de vulnerabilidad es su clara incidencia en la disminución o potenciación de otros factores de vulnerabilidad.

Para finalizar, se puede considerar que la vulnerabilidad, según Chardon (2008, pág. 9) 
[...] corresponde la probabilidad de que un sujeto o elemento expuesto a una amenaza natural, tecnológica, antrópica o socionatural, sufra daños y pérdidas humanas como materiales en el momento del impacto del fenómeno, teniendo además dificultad en recuperarse de ello, a corto, mediano o largo plazo.

\section{A MANERA DE CONCLUSIÓN}

Si bien las construcciones de los conceptos de hábitat y vulnerabilidad son importantes como insumo en la compresión de la realidad, se debe dejar claro que no representan en su totalidad la realidad misma. Se debe abrir camino a entender que la realidad es cambiante, no estática y de difícil predicción, y centrarse en el estudio de las discontinuidades de los procesos históricos que han conformado los hábitats y la vulnerabilidad asociada a estos, superando así la racionalidad instrumental reductora con la que se quiere abordar estos conceptos.

Los modelos de comprensión de la vulnerabilidad y el hábitat pueden ser replanteados de manera que se facilite la interdisciplina. Es posible entender la realidad partiendo de la simplicidad a la complejidad de los fenómenos; combinar en los modelos lo abstracto y lo concreto; abordar los procesos históricos usando métodos inductivos y deductivos; construir conceptos que atiendan la singularidad del conocimiento científico y los saberes populares de las poblaciones sin que sean contradictorios. Todo lo anterior, con el fin de reflexionar sobre los procesos de gestión de hábitats dignos, incluyentes y posibles para los pobladores, que superen las causas de vulnerabilidad y que permitan el bienestar de las poblaciones.

En resumen, el hábitat es el patrón resultante de la relación de las estructuras de naturaleza y sociedad. En el hábitat se contienen las amenazas que hacen vulnerable a las poblaciones; donde la vulnerabilidad se entiende como el resultado de una relación de factores (físico-naturales, socio-económicos, político-institucionales) que la generan mediante un proceso sinérgico y como subsistema del riesgo.

A su vez, la vulnerabilidad determina la probabilidad de que sujetos o elementos expuestos a amenazas (naturales, antrópicas o socio-naturales) sufran daños en vidas u objetos materiales, en el momento del impacto de un fenómeno. La vulnerabilidad se debe considerar antes, durante y después del fenómeno; también representa la aptitud en anticiparse al fenómeno, la capacidad de respuesta, resistencia, adaptación, recuperación y restablecimiento de los medios de vida de las poblaciones que se ven afectadas por un fenómeno.

Finalmente, las anteriores reflexiones permiten mostrar que estos conceptos polisémicos, al igual que la realidad que definen han cambiado y lo seguirán haciendo. No solamente por cuestiones espacio-temporales, sino también, por las personas que viven, construyen hábitats y son vulnerables a diferentes amenazas naturales. Por consiguiente, el trabajo en la conceptualización y contextualización en el hábitat y la vulnerabilidad es fundamental a la hora de generar procesos de gestión en torno a la mejora de la calidad de vida de las poblaciones. 


\section{BIBLIOGRAFÍA}

- Anderson, M. \& Woodrow, P. (1989). Rising from the Ashes: Development Strategies in Times of Desaster. Boulder: Westview Press.

- Ángel Maya, A. (1996). El reto de la vida. Ecosistema y Cultura: Una introducción al estudio del medio ambiente. Bogotá: Editorial Ecofondo.

- Blaikie, P.; Cannon, T.; Davis, I. \& Wisner, B. (1996). Vulnerabilidad. El entorno social, político, y económico de los desastres. Red de Estudios Sociales en Prevención de Desastres en América Latina. La RED.

- Bogotá, S.d. (2011). Misión de la Secretaría del Hábitat. Obtenido de: http://www.bogota.gov.co/portel/libreria/php/01.27090813.html [recuperado el 4 de abril de 2012].

- Bordieu, P. (1997). Capital cultural, escuela y espacio social. Ciudad de México: Siglo XXI Editores.

- Capra, F. (1998). La trama de la vida. Una nueva perspectiva de los sistemas vivos. Barcelona: Editorial Anagrama.

- Cardona, O. (1992). Evaluación de la amenaza, la vulnerabilidad y el riesgo. En: Maskrey, A. Los desastres no son naturales. Bogotá: Red de Estudios Sociales en Prevención de Desastres en América Latina. La RED.

- . (2001). Estimación holística del riesgo utilizando sistemas dinámicos complejos. Tesis Doctoral para optar al título de Ph.D del programa de Ingenería Sísmica y Dinámica. Barcelona: Univerisdad Politenica de Catalunya, Escola Tecnica Superior d'enginyers de camins, canals i ports.

- _ (2003). La necesidad de repensar de manera holística los conceptos de vulnerabilidad y riesgo. Una crítica necesaria para la gestión. Obtenido de: Red de Estudios Sociales en Prevención de Desastres en América Latina http://www.desenredando.org/public/articulos/ 2003/rmhcvr/rmhcvr_may-08-2003.pdf [recuperado el 12 de abril de 2012].

- Chardon, A.-C. (2004). Un enfoque geográfico de la vulnerabilidad global de un hábitat urbano de ladera expuesto a amenazas naturales. El caso andino de Manizales, Colombia. Obtenido de: Human Development Resource Net: http://hdrnet.org/372/1/CHARDON_04.pdf [recuperado el 18 de abril de 2012].

- _ (2008). Amenaza, vulnerabilidad y sociedades urbanas. Una visión desde la dimensión institucional. Revista Gestión y Ambiente, 11(2), 123-136.

- _ (2009). Reasentamiento y poblaciones urbanas vulnerables. Un análisis desde el hábitat, el desarrollo y la sostenibilidad en Manizales, Colombia. Cuadernos de investigación urbanística, 69, 50-70. Simposio de La Serena: Desarrollo, Ciudad y Sostenibilidad.

- H (2012). Hábitat. Conceptos, Acciones, Reflexiones, Propuestas. Obtenido de: Documento Interno Seminario Electivo Hábitat y Vulnerabilidad. Maestría en Hábitat, Escuela de Arquitectura, Universidad Nacional de Colombia sede Manizales. 
- Cundinamarca, D.d. (2004). Lineamientos de Politica de Hábitat. Regional del departamento de Cundinamarca. Bogotá: Ed. Departamento de Cundinamarca.

- Cuny, F. (1983). Disasters and development. New York: Oxford university press. Citado por Maskrey, A. (1998). Navegado entre brumas: La aplicación de los sistemas de información geográfica al análisis del riesgo en América Latina. Lima: LA RED de Estudios Sociales en Prevención de Desastres en América Latina.

- Echavarría, M.C. (2004). Ascensos y descensos de la vivienda: Mirada desde Medellín. Revista INVI, 19(50), 21-69. Instituto de la vivienda. Universidad de Chile.

- _ (2011). Hábitat del habitar, como territorio étnica, grupal y socialmente significado. Obtenido de: Construcción social del hábitat, asunto vital: http://construccionsocialdelhabitat.wordpress.com/ [recuperado el 10 de Marzo de 2012].

- Echavarría, M.C.; Arboleda, E.; Mejía, M.; Cardales, A. y Pérez, A. (2007). Habitar ciudad: Estado del arte en Medellín: 1981-2005. Medellín: Universidad Nacional de Colombia sede Medellín.

- Echevarría, M.C. y Rincón, A. (2000). Análida, Ciudad de Territorialidades: Polémicas de Medellín. Medellín: Centro de Estudios del Hábitat Popular (CEHAP) - Facultad de Arquitectura, Universidad Nacional de Colombia sede Medellín.

- Escobar, A. (2007). La invención del Tercer Mundo. Construcción y deconstrucción del desarrollo. Caracas: Fundación Editorial el Perro y la Rana.

- Fernández, R. (2000). La Ciudad Verde. Teoría de la gestión ambiental urbana. Buenos Aires: Espacio Editorial.

- Fique, L. (2008). Habitát: Hacia un modelo de comprensión. En: Yory, C.M., Pensando en clave de hábitat. Una búsqueda por algo mas que un techo (pp. 102-129). Bogotá: Facultad de Artes, Universidad Nacional de Colombia.

- Foschiatti, A.M. (2012). Vulnerabilidad Global y Pobreza. Consideraciones conceptuales. Obtenido de: Facultad de Humanidades, Universidad Nacional del Nordeste: http://hum.unne.edu.ar/revistas/geoweb/Geo2/contenid/vulner1 .htm

- Giraldo, F. (2003). Hábitat y Sostenibilidad. Colombia. Ciencia y Tecnología, 21(2), 43.

- Gómez de Mantilla, L.T. (2000). Habitar, habitación, habitable, hábito, habitus. Bogotá: Maestría en Medio Ambiente, Universidad Nacional sede Bogotá.

- González, L. (2002). La concepción tecnológica del habitat. Ensayos FORHUM. Miradas al hábitat, 19, 21-33.

- Heidegger, M. (1994). Construir, Habitar, Pensar. Obtenido de: http://ured.manizales.unal.edu.co/modules/uncontextos/ admin/archivos/4050072/habitarpensar.pdf [recuperado el 25 de marzo de 2012].

- Hewitt, K. (1983). The idea of calamity in a technocratic age. London: Allen and Unwin.

- Lampis, A. (2010). Pobreza y riesgo medio ambiental. Obtenido de: La RED de Estudios Latinoamericanos sobre Desastres: www.desenredando.com [recuperado el 24 de marzo de 2012].

- Lavell, A. (1992). Ciencias sociales y desastres naturales en América Latina: Un encuentro inconcluso. Desastres Naturales, Sociedad y Portección Civil. Ciudad de México: COMECSO. 
- Leff, E. (1998). Saber ambiental: sustentabilidad, racionalidad, complejidad, poder. Ciudad de México: Editorial Siglo XXI PNUMA.

- _ (2004). Racionalidad ambiental, la reapropiación social de la naturaleza. Ciudad de México: Siglo XXI Editores.

- Lungo, M. (2002). Expansión urbana y regulación de la tierra en Centroamerica - Antiguos problemas nuevos desafíos. En: PNUD, Riesgos Urbanos (pp. 29-44). San Salvador: Istmo Editores.

- Maskrey, A. (1989). El manejo popular de los desastres naturales. Estudios de vulnerabilidad y mitigación. Lima: ITDG.

- __ (1998). Navegado entre brumas: La aplicación de los sistemas de información geográfica al análisis del riesgo en América Latina. Lima: La RED de Estudios Sociales en Prevención de Desastres en América Latina.

- Medina, J. y Romero, R. (1992). Los deastres sí avisan. Estudios de vulnerabilidad y mitigación II. Lima: ITDG.

- Morin, E. (1995). Introducción al pensamiento complejo. Madrid: Gedisa.

- Noguera, P. (2006). Pensamiento ambiental complejo y gestión del riesgo: Una propuesta epistémico, ético, estética. Manizales: Instituto de Estudios Ambientales - Universidad Nacional de Colombia.

- Ossa, C. (1981). Teoría General de Sistemas. Problemas teóricos y prácticos. Mérida, Venezuela: Publicación interna EISULA.

- Quarantelli, H. (1987). What should we study? Questions and suggestions for researchers about the concepts of disasters. International Journal of mass emergencies and disasters, 5(1), 732.

- _ (2001). Urban vulnerability to disaster on developing countries: Managin risk. Obtenido de: http://www.bvsde.paho.org/bvsacd/cd46/cap15-urban2.pdf. [recuperado el 2 de marzo de 2012].

- Ratick, S. (1994). Coping with Climate Change: Vulnerability and Response to Sea level Rise and Severe Storms. Worcester: Center for Technology, Environment and Development. Clark University.

- Romero, G. y Maskrey, A. (1993). Cómo entender los desastres naturales. En: Maskrey, A., Los desastres no son naturales. Bogotá: Red de Estudios Sociales en Prevención de Desastres en América Latina, La RED.

- Rubio, J. (1993). Modelos y mensajes. Bogotá: Ed. Significantes de Papel.

- Santos, M. (2000). La naturaleza del Espacio. Técnica y tiempo. Razón y emoción. Barcelona: Ariel.

- Susman, P.; O'Keefe, P. \& Wisner, B. (1984). Global Disasters, a Radical Interpretation. In: Hewitt, K., Interpretations of Calamity: From the Viewpoint of Human Ecology (The Risks \& Hazards Series, 1) (pp. 264-283). Winchester, Mass.: Allen \& Unwin.

- Turner, B.; Kasperson, R.; Matson, P.; McCarthy, J.; Corell, R.; Christensen, L. et al. (2003). A framework for vulnerability analysis in sustainability science. PNAS Review., 100(14), 8074-8079. Obtenido de: http://www.pnas.org/content/100/14/8074.full.pdf [recuperado el 3 de mayo de 2012].

- UN-HABITAT. (2009). Origen del UN-HABITAT. Obtenido de: http://www.onuhabitat.org/index.php?option=com_content\& view=article $\& i d=72 \&$ Itemid $=85$ 

(2012). Hábitat y Desarrollo Humano. Cuadernos PNUDUN Hábitat. Colombia: Panamericana Formas e Impresos S.A.

- Vidal, R. (2002). La utopía, después del "fin de las utopías". Pensar en un futuro abierto más alla del progreso. Obtenido de: Espéculo. Revista electrónica de estudios literarios de la Facultad de Ciencias de la Información de la Universidad Complutense de Madrid, 22 (1): http://www.ucm.es/info/especulo/numero22/utopia.html [recuperado el 18 de enero de 2009].

- Westgate, K. \& O'keefe, P. (1976). Some definitions of disaster. Ocasional Paper No. 4. Disaster research unit, 69.

- Wilches-Chaux, G. (1989). La vulnerabilidad global. En: Desastres, Ecologismo y Formación Profesional: Herramientas para la Crisis. Popayán: Servicio Nacional de Aprendizaje -SENA-.

Para citar este artículo: Jiménez, W. G. (2013). Hábitat y vulnerabilidad, reflexiones desde lo conceptual. Revista Luna Azul, 37, 196-218. Recuperado de http://lunazul.ucaldas.edu.co/index.php?option=content\&task=view\&id=852 